\title{
Comment on "Bilirubin: a novel predictor of hemorrhagic transformation and symptomatic intracranial hemorrhage after mechanical thrombectomy"
}

\author{
Erkan Cure $^{1} \cdot$ Medine Cumhur Cure ${ }^{2}$ \\ Published online: 8 January 2020 \\ C) Fondazione Società Italiana di Neurologia 2020
}

Dear Sir,

We read with great pleasure the very important study evaluating "Bilirubin (B): a novel predictor of hemorrhagic transformation (HT) and symptomatic intracranial hemorrhage (ICT) after mechanical thrombectomy" by Jian et al. [1]. We would like to draw attention to several important points in this significant study.

Can blood B levels be a predictive marker of the severity of ICT? Their high B levels in HT patients were higher than those of the without HT (WHT) group, suggesting that B is an important marker for both mortality and morbidity in ICT. After ICT occurs, erythrocytes begin to undergo hemolysis within minutes as the body's self-defense mechanism against acute brain damage [2]. Firstly, heme is released from erythrocytes by the hemolysis, then the heme is cleaved by hem oxygenase enzyme and as a result of this degradation, biliverdin, iron, and carbon monoxide occurs. Iron is a highly potent toxic agent for the brain and initiates Fenton reaction and lipid peroxidation [2]. It shows the neurotoxic effect by increasing superoxide and hydroxyl radicals and oxidative stress (OS). The resulting carbon monoxide and raised reactive oxygen radicals cause severe damage to the brain. Biliverdin reductase enzyme activity in the brain has been shown, and its deficiency has been associated with Alzheimer's disease. Biliverdin, which is the product of heme destruction, is converted to B by biliverdin reductase enzyme. Normally, the blood-brain

Erkan Cure

erkancure@yahoo.com

Medine Cumhur Cure

medinecure@yahoo.com

1 Department of Internal Medicine, Ota\&Jinemed Hospital, Muradiye Mahallesi Nuzhetiye Cad, Deryadil Sokagi No:1, 34357 Besiktas, Istanbul, Turkey

2 Department of Biochemistry, Istanbul, Turkey barrier (BBB) is permeable to lipophilic substances. B in the blood can slowly and easily pass through the brain, as it is bound on albumin due to hyperbilirubinemia. Hypoxia and raised OS may disrupt the protective permeability features of the BBB and cause the passage of neurotoxic substances into the brain [3]. As a result of this severe brain injury, B can slowly pass through the abluminal surface of the BBB to the systemic circulation. A portion of the $\mathrm{B}$ is bound to the albumin and forms the direct $B$, while the other part is not bound to proteins and is identified as indirect $B$. We speculate that $B$ may occur with hemolysis of erythrocytes in the bleeding area during ICT and this B in the brain can easily move through into the systemic circulation with increased permeability of BBB by hypoxia and oxidative stress damages. B levels in their ICT patients were slightly higher than the WCT group. The slight elevation in B may be due to cerebral hemoglobin hemolysis.

Could elevated B levels in their HT patients than the WHT group be due to other causes? $\mathrm{B}$ is a potent antioxidant and has a strong cardioprotective effect [4]. Both indirect and direct B levels have been reported to reduce atherosclerosis and OS. However, in recent publications, it has been reported that B elevation is related to arrhythmia, especially, high total B levels have an association with atrial fibrillation $(\mathrm{AF})$ [5]. In the Jian et al. study, the number of AF patients in the HT group was 2.5 times more than in the WHT group [1]. Therefore, the high B levels in the HT group may be due to the high number of AF patients. Heart failure (HF) is also associated with increased $\mathrm{B}$ levels. The HF incidence is increased in patients with AF. It is not clear whether patients with $\mathrm{HF}$ are included in their study [1]. Calorie restriction (400-calorie diet for $24 \mathrm{~h}$ ) and prolonged fasting are known to increase B levels. In the Jian et al. study, the fasting duration of the patients on admission in the study was not clear. Also, it is unclear whether their patients have calorie restriction. Further studies are needed to investigate the high B levels in HT patients. 


\section{Compliance with ethical standards}

Conflict of interest The authors declare that there is no conflict of interest regarding the publication of this article.

\section{References}

1. Jian Y, Zhao L, Wang H (2019) Bilirubin: a novel predictor of hemorrhagic transformation and symptomatic intracranial hemorrhage after mechanical thrombectomy. Neurol Sci:1-7. https://doi.org/10. 1007/s10072-019-04182-x

2. Righy C, Bozza MT, Oliveira MF, Bozza FA (2016) Molecular, cellular and clinical aspects of intracerebral hemorrhage: are the enemies within? Curr Neuropharmacol 14:392-402
3. Lochhead JJ, McCaffrey G, Quigley CE et al (2011) Oxidative stress increases blood-brain barrier permeability and induces alterations in occludin during hypoxia-reoxygenation. J Cereb Blood Flow Metab 30(9):1625-1636. https://doi.org/10.1038/jcbfm.2010.29 Erratum in: J Cereb Blood Flow Metab 31:790-791

4. Cure MC, Cure E, Kirbas A et al (2013) The effects of Gilbert's syndrome on the mean platelet volume and other hematological parameters. Blood Coagul Fibrinolysis 24:484-488. https://doi.org/10. 1097/MBC.0b013e32835e4230

5. Chen SC, Chung FP, Chao TF, Hu YF, Lin YJ, Chang SL, Lo LW, Tuan TC, te ALD, Yamada S, Prabhu A, Chang TY, Lin CY, Chen SA (2019) A link between bilirubin levels and atrial fibrillation recurrence after catheter ablation. J Chin Med Assoc 82:175-178. https://doi.org/10.1097/JCMA.0000000000000026

Publisher's note Springer Nature remains neutral with regard to jurisdictional claims in published maps and institutional affiliations. 